\title{
Single-Molecule FRET Combined with Magnetic Tweezers at Low Force Regime
}

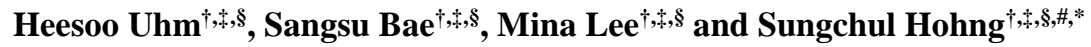 \\ Department of Physics and Astronomy, Institute of Applied Physics, ${ }^{8}$ National Center of Creative Research Initiatives, \\ ${ }^{\#}$ Department of Biophysics and Chemical Biology, Seoul National University, Seoul 151-747, Korea \\ "E-mail: shohng@snu.ac.kr \\ Present address : Sangsu Bae(Department of Chemistry, Hanyang University, Seoul 133-791, Korea), Mina Lee(Center for \\ Nano-Bio Measurement, Korea Research Institute of Standards and Science, Daejeon 305-600, Korea) \\ Received November 12, 2015, Accepted Date (to be inserted by publisher after your manuscript is accepted)
}

Keywords : Magnetic tweezers, single molecule FRET, Holliday junction

Mechano-sensitive reactions are ubiquitously observed during DNA replication, RNA transcription, cell movements and the operation of transport proteins. ${ }^{1}$ In order to study these reactions at the single-molecule level, various singlemolecule manipulation techniques such as atomic force microscopy, optical tweezers, and magnetic tweezers (MT) have been developed, ${ }^{2}$ and these techniques have been actively used to study a variety of biochemical reactions which are sensitive to mechanical stresses.

In biology, forces as small as in the sub-piconewton range can cause dramatic effects on the kinetics of biochemical reactions. The sole mechanical manipulation tools, however, suffer from poor spatial resolution at the small force regime, and this technical limitation has hindered the study of subtle conformational changes induced by small forces. To solve the problem, several research groups tried to combine single-molecule manipulation techniques with single- molecule fluorescence resonance energy transfer (FRET). ${ }^{3-8}$ In this approach, force-sensitive conformational changes as small as in the sub-nanometer range could be reliably monitored using single-molecule FRET.

Magnetic tweezers have a few advantages over other single-molecule manipulation techniques; small forces can be reliably applied by adjusting the height of a magnet, and twisting stress can be easily introduced by rotating the magnet. ${ }^{4}$ Recently, magnetic tweezers were combined with single-molecule FRET by several groups and proven to be useful for measuring conformational changes of biomolecule in the nanometer range. ${ }^{4,6}$ In their work, however, it was not clear what the low force limit is that can be reliably used for the combined measurements of FRET and forces.

Here, we developed a single-molecule FRET microscope combined with MT setup and characterized the capabilities of the instrument. Especially, we clarified the low force limit
(A)

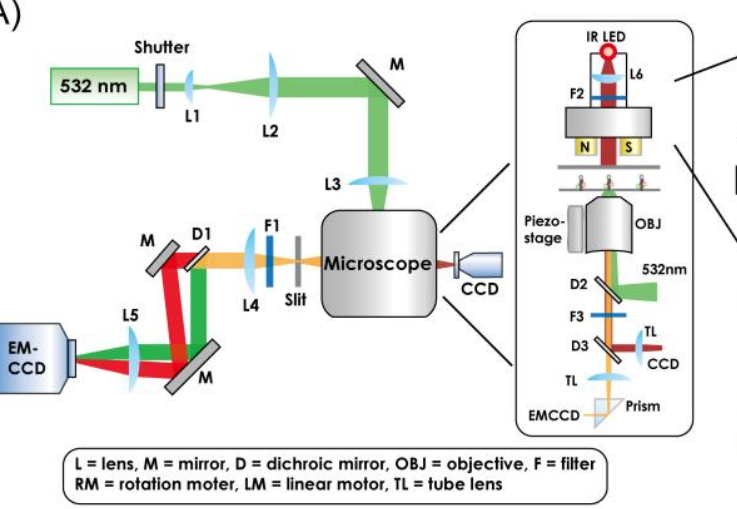

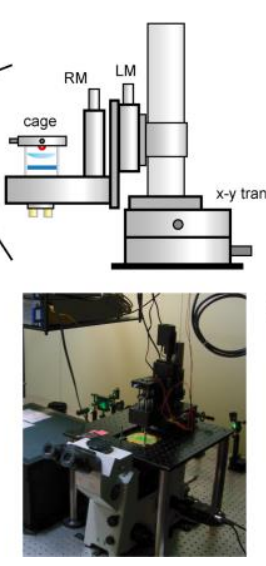

(B)

(D)
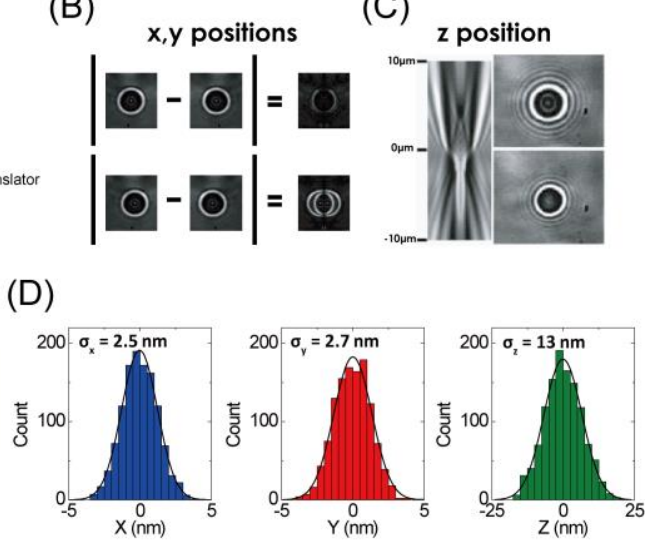

Figure 1. (A) Schematic diagram of the FRET-MT experimental setup. The identities of the components are: L1, a lens ( $\mathrm{f}=25.4 \mathrm{~mm}$, LA1951-A, Thorlabs); L2, a lens $(\mathrm{f}=250 \mathrm{~mm}$, LA1301-A, Thorlabs); L3, an achromatic lens $(\mathrm{f}=300$ $\mathrm{mm}$, AC508 300A, Thorlabs); L4, an achromatic lens ( $\mathrm{f}=120 \mathrm{~mm}, 01$ LAO 538, Melles Griot); L5, an achromatic lens ( $\mathrm{f}=260.1 \mathrm{~mm}, 01 \mathrm{LAO}$ 638, Melles Griot); L6, a lens ( $=50 \mathrm{~mm}$, LA1131-A, Thorlabs); D1, a dichroic mirror (640dcxr, Chroma); D2, a dichroic mirror (z532rdc, Chroma); D3, a dichroic mirror (z780dcspxr, Chroma); F1, a shortpass filter (HQ750sp-2p, Chroma); F2, a bandpass filter (HQ850/90x, Chroma); F3, a longpass filter (LP03532RU, Chroma). (B) Symmetry recognition method for xy positioning. (C) Radial intensity profiles for z positioning and images of the bead with two different heights. (D) Histograms of $\mathrm{x}, \mathrm{y}$ and $\mathrm{z}$ positions of a bead immobilized on the coverglass. 


\section{BULLETIN OF THE KOREAN CHEMICAL SOCIETY}

H. Uhm et al.

positioning was tested using stuck beads. The standard deviations of the bead position were $2.5 \sim 2.7 \mathrm{~nm}$ in the $\mathrm{x}-\mathrm{y}$ direction, and $13 \mathrm{~nm}$ in the $\mathrm{z}$ direction (Fig. 1D). The result is comparable to the previously reported results. ${ }^{10}$

To perform single-molecule experiments, we made a DNA construct composed of a Holliday junction at the bottom and a $\lambda$ phage DNA at the top (Fig. 2A, Experimental Section). The Holliday junction is composed of four helical arms of double-stranded DNA. ${ }^{11}$ It is known that the Holliday junction dynamically switches between two distinct conformations. ${ }^{9}$ To monitor the small conformational dynamics using single-molecule FRET, two arms of the Holliday junction were labeled with FRET probes at the ends. The bottom part of the DNA was immobilized on a glass surface using the biotin-streptavidin interaction. The top part of the DNA was connected to a magnetic bead using the digoxigenin-antidigoxigenin interaction. The force-extension curve of the DNA was nicely fitted to a worm-like chain model with $\sim 16 \mu \mathrm{m}$ DNA length and $\sim 30 \mathrm{~nm}$ persistence length (Fig. 2B), which is comparable to the previously reported value. ${ }^{12}$

Magnetic beads generate auto-fluorescence in the visible region, which can easily overwhelm the single-molecule fluorescence signals when they are positioned near the glass surface (Fig. 2C). We measured the auto-fluorescence of magnetic beads as a function of the bead height. The autofluorescence intensity exponentially decreased with the bead height, and became negligible above $2 \mu \mathrm{m}$ compared to single-molecule fluorescence signals (Fig. 2D). This height corresponds to $0.05 \mathrm{pN}$ when $\lambda$ DNA was used as a linker. At low force regime, the end-to-end distance of DNA duplex is much shorter than its contour length. The result above indicates that DNA duplexes much longer than $2 \mu \mathrm{m}$ should diffraction pattern of a bead with the calibration images (Fig. $1 \mathrm{C}$, Experimental Section). ${ }^{10}$ The accuracy of the bead

(A)

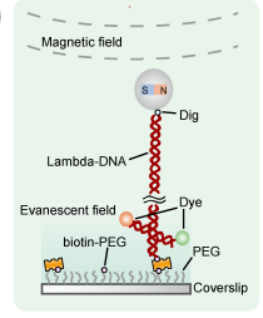

(C)

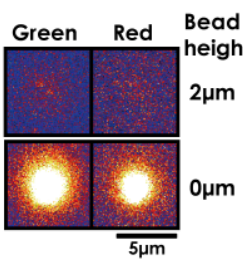

(B)

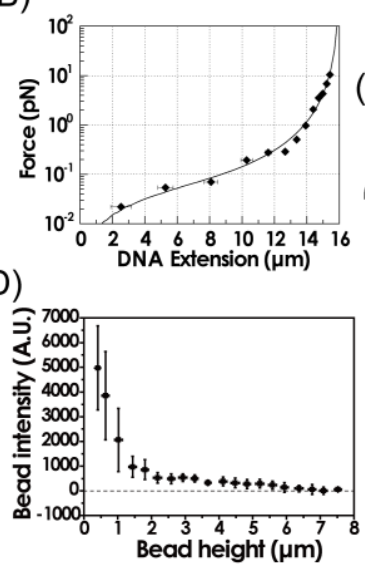

$(\mathrm{E})$

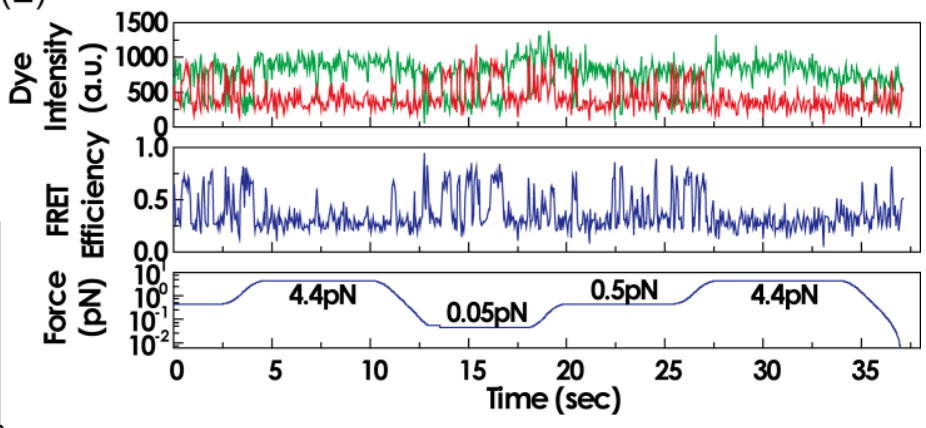

Figure 2. (A) Schematic diagram of the FRET-MT experiment for the Holliday junction. (B) Force-extension curve of the DNA sample. The solid line represents the fit to the wormlike chain (WLC) model. (C) Donor and acceptor channels recorded by EMCCD. Green laser excitation is always on. Bead height is about $2 \mu \mathrm{m}$ (top) or $0 \mu \mathrm{m}$ (bottom). (D) Intensity of bead autofluorescence at varying heights. (E) Force-dependent conformational dynamics of the Holliday junction observed via FRET. 
be used to study the effects of low forces.

It is known that the conformational dynamics of the Holliday junction is sensitive to mechanical forces applied to its helical arms. While applying varying forces to the Holliday junction using magnetic tweezers, we observed the conformational dynamics of the Holliday junction (Fig. 2E). We could observe the two-state dynamics of the Holliday junction with a good signal-to-noise ratio even at $0.05 \mathrm{pN}$. At higher forces, the Holliday junction dynamics were biased to the low-FRET state as previously reported. ${ }^{9}$

In conclusion, we made a FRET-MT setup, and characterized that the low force limit of the combined measurements was $\sim 0.05 \mathrm{pN}$. With the development of the new microscope, it is expected that mechanochemical studies at the single-molecule level will become applicable to the studies of wider biomolecular processes.

\section{Experimental Section}

FRET-MT setup. The instrument was made by modifying a commercial inverted fluorescence microscope (Olympus, IX71). The fluorescence excitation laser was guided through the back-port of the microscope, whereas fluorescence signals were guided through the left side port of the microscope to an EM-CCD camera (Andor, iXon+). ${ }^{13}$ The magnetic tweezers were placed on top of the microscope, and magnetic beads were imaged using a CCD camera on the right side port. The $\mathrm{x}$ position of beads was determined analyzing the sum of absolute difference $(\mathrm{SAD}=$ $\left.\Sigma_{\mathrm{i}, \mathrm{j}}|\mathrm{I}(\mathrm{i}, \mathrm{j})-\mathrm{I}(-(\mathrm{i}-\Delta), \mathrm{j})|\right)$ between the original image(I(i,j)) and the reversed image $(\mathrm{I}(-\mathrm{i}, \mathrm{j}))$ at varying $\Delta$. The SAD value becomes smallest when the two images overlap. The half of $\Delta$ that minimizes SAD was determined as the x symmetry center position of $I(i, j)$. The y position was determined using the same method, only swapping $\mathrm{i}$ and $\mathrm{j}$. For $\mathrm{z}$ positioning, the radial intensity profiles of a stuck bead were collected for the heights in the $20 \mu \mathrm{m}$ range by a piezoelectric objective scanner (Physik Instrumente, P-721), and the data were used for the comparison with the measured bead image. ${ }^{10}$ To obtain the force calibration curve as a function of the magnet position, time trajectories of the $x y$ position of magnetic beads were obtained at varying magnet positions. By analyzing the power spectra of the trajectories, the force at specific magnet position was determined, ${ }^{10}$ and the calibration data were fitted to a linear function on a semi-log scale. ${ }^{14}$ During experiments, the fitting curve was used to determine the magnet position to apply the specified forces to DNA constructs. ${ }^{14}$

Sample preparation and experiments. $\lambda$ DNA was purchased from NEB. Short DNA oligonucleotides were purchased from IDT-DNA. The sequences of the DNA strands comprising the Holliday junction are: b-strand (5'/Cy5/CCCTAGCAAGCCGCTGCTACGG-3'), h-strand (5' -

\section{H. Uhm et al.}

/Cy3/CCGTAGCAGCGCGAGCGGTGGG-3'), r-strand (5' /biotin/CCCACCGCTCGGCTCAACTGGG-3'), x-strand (5'-GGGCGGCGACCTCCCAGTTGAGCGCTTGCTAG GG-3'). A short oligo (Dig-oligo: 5'-AGGTCGCCGCCC /digoxigenin/-3') was used to connect $\lambda$ DNA to an antidigoxigenin coated bead (Invitrogen, Dynal magnetic bead, $2.8 \mu \mathrm{m})$. The Holliday junction- $\lambda$ DNA construct and antidigoxigenin coated beads were prepared as previously reported. ${ }^{9}$ The Holliday junction- $\lambda$ DNA constructs were immobilized on a streptavidin-coated glass surface of the detection channel. To make DNA-bead constructs, antidigoxigenin coated beads diluted in a buffer containing $10 \mathrm{mM}$ Tris ( $\mathrm{pH} 8.0$ ) and $50 \mathrm{mM} \mathrm{MgCl}_{2}$ were delivered into the detection channel. Before starting experiments, an imaging buffer (10 mM Tris ( $\mathrm{pH} 8.0), 0.4 \mathrm{mg} / \mathrm{ml}$ Trolox, $0.4 \%$ (w/v) glucose, $1 \mathrm{mg} / \mathrm{ml}$ glucose oxidase, $0.02 \mathrm{mg} / \mathrm{ml}$ catalase and $50 \mathrm{mM} \mathrm{MgCl} 2$ ) was injected to the detection channel.

Acknowledgments. We would like to thank O. Saleh and K. Kim at the University of California, Santa Barbara for generous advices about building magnetic tweezers. This work was supported by Creative Research Initiative (20090081562).

\section{References}

1. Hoffman, B. D.; Grashoff, C.; Schwartz, M. A. Nature 2011, 475, 316 DOI: 10.1038/nature10316.

2. Neuman, K. C.; Nagy, A. Nat. Methods 2008, 5, 491 DOI: $10.1038 /$ nmeth. 1218 .

3. Hohng, S.; Lee, S.; Lee, J.; Jo, M. H. Chem. Soc. Rev. 2014, 43, 1007 DOI: 10.1039/C3CS60184F.

4. Lee, M.; Kim, S. H.; Hong, S.-C. Proc. Natl. Acad. Sci. 2010, 107, 4985 DOI: 10.1073/pnas.0911528107.

5. Bae, W.; Kim, K.; Min, D.; Ryu, J.-K.; Hyeon, C.; Yoon, T.-Y. Nat. Commun. 2014, 5, 5654 DOI: 10.1038/ncomms6654.

6. Long, X.; Parks, J. W.; Bagshaw, C. R.; Stone, M. D. Nucleic Acids Res. 2013, 41, 2746 DOI: 10.1093/nar/gks1341.

7. Lee, S.; Hohng, S. J. Am. Chem. Soc. 2013, 135, 18260 DOI: $10.1021 /$ ja408767p.

8. He, Y.; Lu, M.; Cao, J.; Lu, H. P. ACS Nano 2012, 6, 1221 DOI: $10.1021 / \mathrm{nn} 2038669$.

9. Hohng, S.; Zhou, R.; Nahas, M. K.; Yu, J.; Schulten, 
K.; Lilley, D. M. J.; Ha, T. Science 2007, 318, 279 DOI: $10.1126 /$ science.1146113.

10. Gosse, C.; Croquette, V. Biophys. J. 2002, 82, 3314 DOI: 10.1016/S0006-3495(02)75672-5.

11. Grainger, R. J.; Murchie, A. I. H.; Lilley, D. M. J. Biochemistry (Mosc.) 1998, 37, 23 DOI: 10.1021/bi9721492.

12. Baumann, C. G.; Smith, S. B.; Bloomfield, V. A.; Bustamante, C. Proc. Natl. Acad. Sci. 1997, 94, 6185.

13. Roy, R.; Hohng, S.; Ha, T. Nat. Methods 2008, 5, 507 DOI: 10.1038/nmeth.1208.

14. Lipfert, J.; Hao, X.; Dekker, N. H. Biophys. J. 2009, 96, 5040 DOI: 10.1016/j.bpj.2009.03.055. 
Note

BULLETIN OF THE

www.bkcs.wiley-vch.de

KOREAN CHEMICAL SOCIETY

H. Uhm et al.

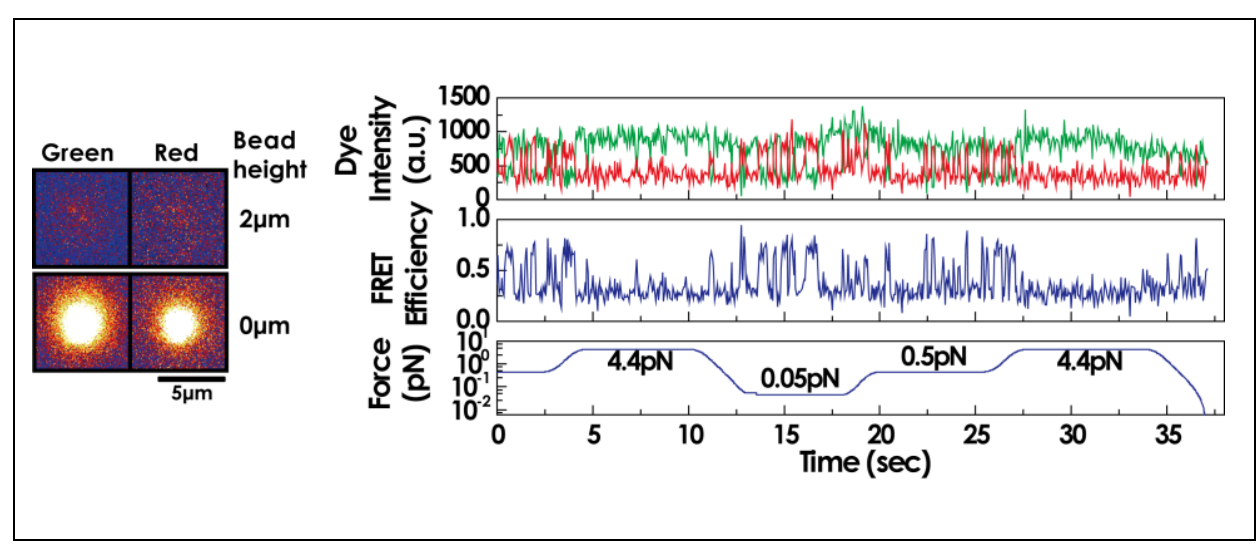

Graphical Abstract 